\title{
Breakdown of the Chiral Anomaly in Weyl Semimetals in a Strong Magnetic Field
}

\author{
Pilkwang Kim, Ji Hoon Ryoo, and Cheol-Hwan Park* \\ Department of Physics, Seoul National University, Seoul 08826, Korea
}

(Dated: December 29, 2017)

\begin{abstract}
The low-energy quasiparticles of Weyl semimetals are a condensed-matter realization of the Weyl fermions introduced in relativistic field theory. Chiral anomaly, the nonconservation of the chiral charge under parallel electric and magnetic fields, is arguably the most important phenomenon of Weyl semimetals and has been explained as an imbalance between the occupancies of the gapless, zeroth Landau levels with opposite chiralities. This widely accepted picture has served as the basis for subsequent studies. Here we report the breakdown of the chiral anomaly in Weyl semimetals in a strong magnetic field based on ab initio calculations. A sizable energy gap that depends sensitively on the direction of the magnetic field may open up due to the mixing of the zeroth Landau levels associated with the opposite-chirality Weyl points that are away from each other in the Brillouin zone. Our study provides a theoretical framework for understanding a wide range of phenomena closely related to the chiral anomaly in topological semimetals, such as magnetotransport, thermoelectric responses, and plasmons, to name a few.
\end{abstract}

Although Weyl fermions or chiral fermions are basic building blocks for relativistic theories, there are no known massless fermions with a definite chirality among the elementary particles in nature since neutrinos were found to be massive. Very recently, however, it was found that some condensed-matter systems, so-called Weyl semimetals, host Weyl fermions as low-energy quasiparticles [1. Weyl semimetals have Weyl points in the Brillouin zone around which the energy versus momentum relation is linear. Shortly after theoretical predictions in 2015 2, 3, TaAs, NbAs, TaP, and NbP have been confirmed to be Weyl semimetals by angle-resolved photoemission spectroscopy experiments [4-8].

The chiral anomaly, which is relevant to bulk transport [9], is one of the two most intriguing phenomena of Weyl semimetals, the other being the Fermi arcs appearing in the surface electron spectrum [1]. In relativistic field theory, chiral anomaly refers to the nonconservation of chiral charge in the presence of parallel electric and magnetic fields and was first introduced to explain the two-photon decay of the neutral pion [10, 11].

In 1983, Nielsen and Ninomiya predicted that the chiral anomaly in condensed-matter systems manifests itself as negative longitudinal magnetoresistance [9]. This prediction is based on the characteristic linear energy-vswavevector dispersions of the chiral zeroth Landau levels (ZLLs). An electric field applied parallel to the magnetic field induces the charge imbalance between the ZLLs with opposite chiralities (Figs. 1a and 1c). Although the chiral ZLLs may or may not intersect with each other depending on how the two Weyl points align with the magnetic field (Figs. 1a and 1b), the explanation by Nielsen and $\mathrm{Ni}-$ nomiya [9] is valid in all cases (Figs. 1c and 1d). All theoretical and experimental studies on the phenomena in Weyl semimetals related to the chiral anomaly are based on this traditional picture [12 25]. Especially, the negative longitudinal magnetoresistance that was originally suggested by Nielsen and Ninomiya [9] has been observed in many experiments and interpreted as a signature of the chiral anomaly in Weyl semimetals [18, 20].

In this paper, we report the breakdown of the chiral anomaly in Weyl semimetals in a strong magnetic field based on comprehensive ab initio calculations on the Landau levels of TaAs, NbAs, TaP, and NbP. In particular, we find that an energy gap may open up due to the mixing of the ZLLs associated with the Weyl points with opposite chiralities that are separated in the Brillouin zone. The size of the energy gap strongly varies with the strength and direction of the applied magnetic field.

We first consider the Landau levels of TaAs in a magnetic field along $x$ (see Supplementary Figs. 1 and 2 for the crystal structure and the electronic band structure, respectively [26]). The energy-vs-wavevector relation of Landau levels is dispersive only along the direction of the applied magnetic field. There are four positive $k_{x}$ values at which the Landau-level states are associated with the Weyl points of TaAs (A, B, C, and D in Fig. 2a). In a relatively weak magnetic field $(B \leq 10 \mathrm{~T}$ where $B$ is the strength of the magnetic field), the seemingly gapless ZLLs are clearly visible near A, B, and C (Figs. 2b and $3 \mathrm{a}-3 \mathrm{c})$.

Remarkably, energy gaps open up at the crossing of the ZLL energy bands around A and B as the magnetic field increases (Figs. 2c, 2d, 3d, and 3e). The gapped ZLLs are simultaneously associated with the two Weyl points of opposite chiralities that are separated in the Brillouin zone. The charge carriers occupying the gapped ZLLs are therefore not chiral.

We emphasize that this gap opening is totally different from the previously reported gap opening in Dirac semimetals [27-29]. It was found that an external magnetic field that breaks the rotational symmetry protecting the Dirac semimetallic phase induces an energy gap in the ZLLs of a Dirac semimetal [27 29]. However, according to those studies, the ZLLs of a Weyl semimetal should remain gapless. 
a

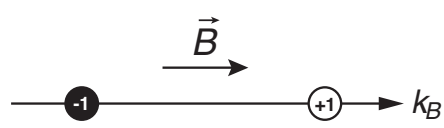

b

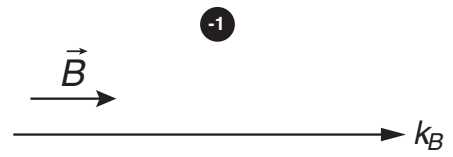

$(1)$

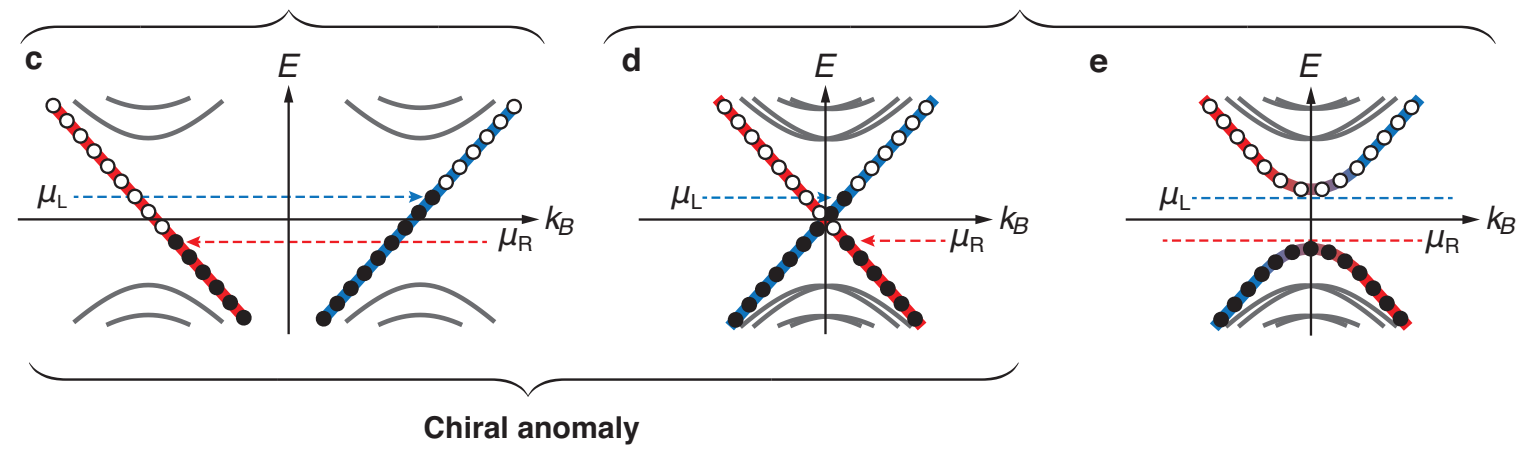

FIG. 1. Chiral anomaly in Weyl semimetals and its breakdown. a and b, Schematics of Weyl points in a magnetic field. $k_{B}$ is the wavevector along the direction of the magnetic field. Encircled numbers are the chiral charges of the Weyl points. Two Weyl points with opposite chiralities lie along a line parallel (a) or perpendicular (b) to the magnetic field. c, A schematic of the Landau-level spectrum of Weyl fermions with chirality +1 and -1 in the presence of parallel electric and magnetic field. Occupied and unoccupied states are shown as black and white discs, respectively. $\mu_{\mathrm{L}}$ and $\mu_{\mathrm{R}}$ are the chemical potentials of the left and right electrodes and are associated with the charge carriers moving to the right and to the left, respectively. Here, the separation of the two Weyl points along $k_{B}$ is large (a). Note that the populations of the Weyl fermions with chirality +1 and -1 are different, which is chiral anomaly. $\mathbf{d}$, The same schematic as in $\mathbf{c}$ but for a case with a small separation in $k_{B}$ between the Weyl points (b). As in $\mathbf{c}$, the populations of the Weyl fermions with chirality +1 and -1 are different. e, A similar schematic as in $\mathbf{d}$ but for a case with an energy gap. Here the chiral anomaly breaks down.
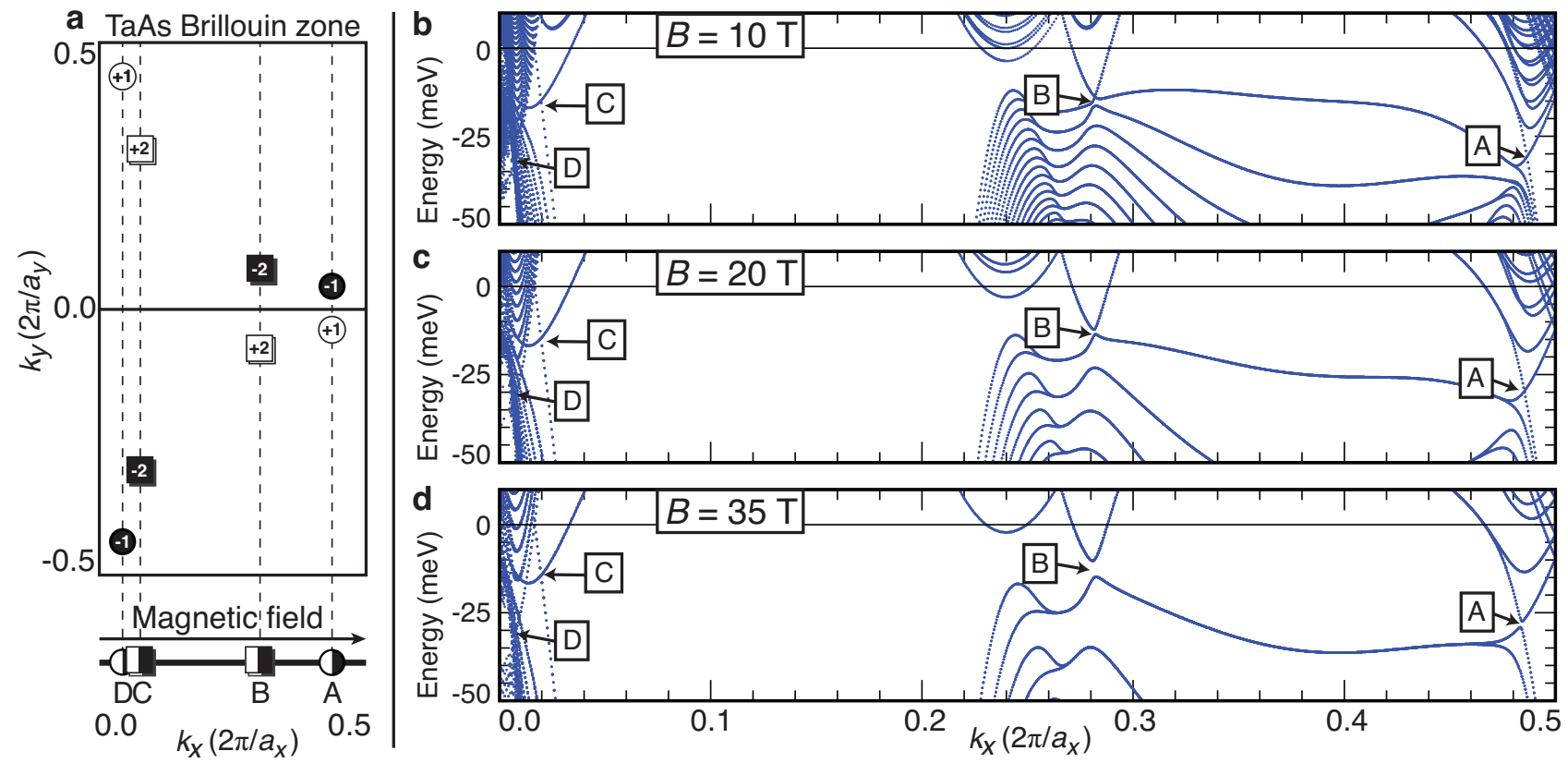

FIG. 2. Landau levels of TaAs. a, A schematic (not to scale) of the projected Weyl points and their chiral charges (the enclosed numbers). Half of the Brillouin zone $\left(k_{x}>0\right)$ is shown. Discs and squares represent two different classes of Weyl points. b-d, Landau-level spectra of TaAs in a magnetic field applied along $x$ obtained from $a b$ initio tight-binding calculations. The zero of energy is set at the Fermi level before the application of the magnetic field. A, B, C, and D denote the corresponding Weyl points shown in a. 


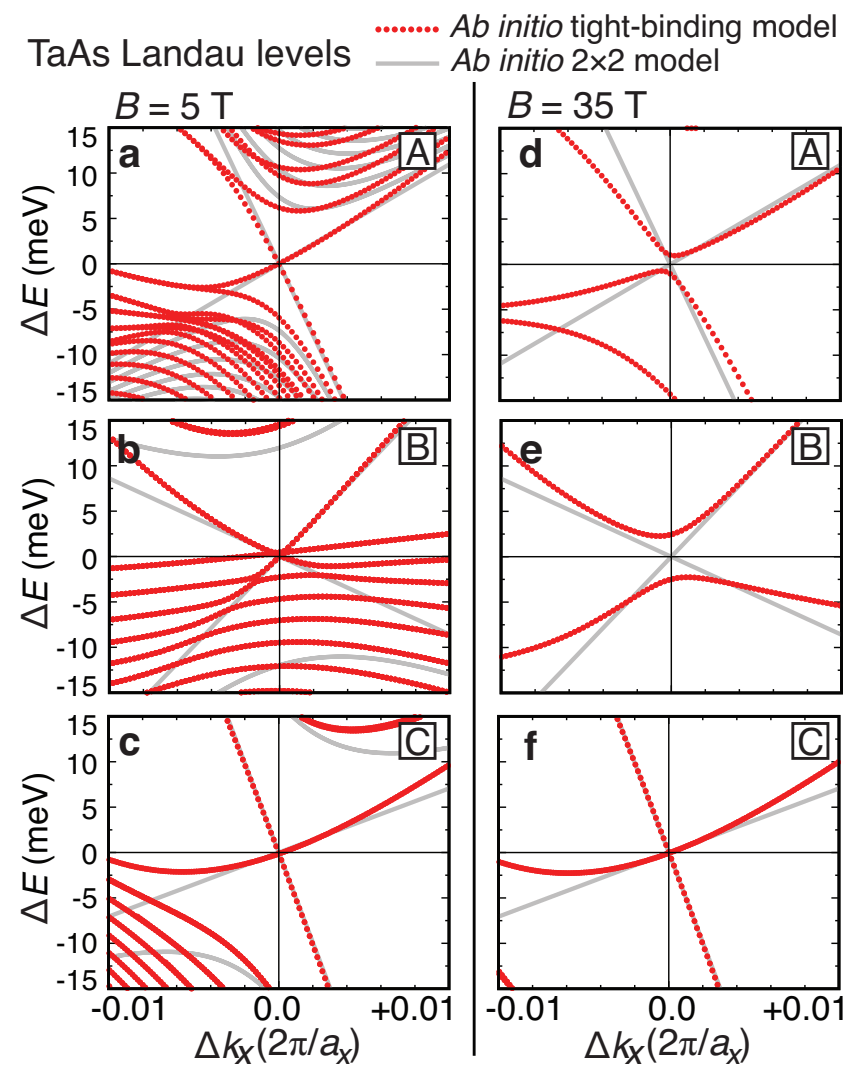

FIG. 3. Magnetic-field-induced gap opening in TaAs. Landau level spectra around A (a and $\mathbf{d}), \mathrm{B}$ ( $\mathbf{b}$ and $\mathbf{e})$, and $\mathrm{C}$ (c and f), at $B=5 \mathrm{~T}(\mathbf{a}-\mathbf{c})$ and $B=35 \mathrm{~T}(\mathbf{d}-\mathbf{f})$. Red discs and solid grey curves show the results obtained from the $a b$ initio tight-binding model and those obtained from the $a b$ initio $2 \times 2$ model, respectively. The zero of energy is set at the mid-point of the higher- and lower-energy zeroth Landau levels.

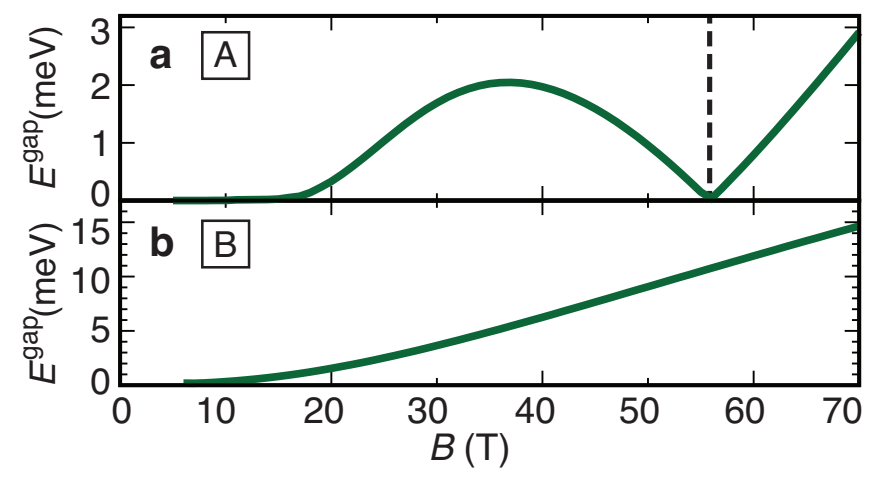

FIG. 4. Band inversion and non-trivial dependence of the energy gap on the strength of the magnetic field. $\mathbf{a}$ and $\mathbf{b}$, The energy gap $E^{\text {gap }}$ of the zeroth Landau levels near A (a) or B (b) versus the strength of the magnetic field. The dashed vertical line in $\mathbf{a}$ indicates the magnetic field at which the higher- and lower-energy zeroth Landau levels are inverted.
TaAs Landau levels $(B=35 \mathrm{~T})$
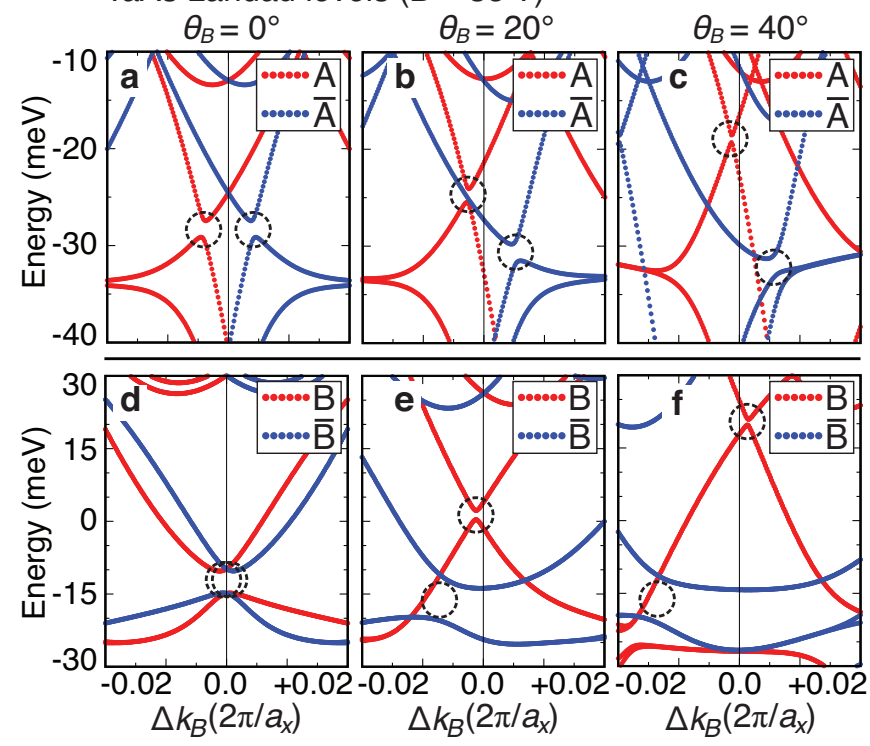

FIG. 5. Dependence of the Landau levels of TaAs on the direction of the magnetic field. Landau-level spectra at $B=35 \mathrm{~T}$. The $k_{x}$ component of the origin is set at that of the corresponding Weyl point: A or its complement $\overline{\mathrm{A}}$, i.e., $\mathbf{k}_{\overline{\mathrm{A}}}=-\mathbf{k}_{\mathrm{A}}(\mathbf{a}-\mathbf{c})$, or $\mathrm{B}$ or its complement $\overline{\mathrm{B}}(\mathbf{d}-\mathbf{f})$ (see Supplementary Fig. 1c [26]), while the $k_{y}$ component of the origin is set at zero. The zero of energy is set at the energy of the corresponding Weyl point in the absence of an external magnetic field. The magnetic field is applied in the $x y$ plane. The angle between the magnetic field and the $x$ axis, $\theta_{B}$, is $0^{\circ}$ ( $\mathbf{a}$ and $\mathbf{d}$ ), $20^{\circ}$ ( $\mathbf{b}$ and $\mathbf{e}$ ), and $40^{\circ}$ (c and $\mathbf{f}$ ). $\Delta k_{B}$ is the distance from the origin of the wavevector along the direction parallel to the applied magnetic field.

The size of these energy gaps depends on the strength of the magnetic field in a non-trivial way. In the case that $B \leq 10 \mathrm{~T}$, the energy gaps are finite but smaller than $0.1 \mathrm{meV}$ (Fig. 4). The energy gap increases with the magnetic field and could become larger than $10 \mathrm{meV}$ at $B=35 \mathrm{~T}$ (Figs. 4 and 5). As the magnetic field further increases, the size of the energy gap of the ZLLs near A decreases, becomes zero, and then increases again (Fig. 4a). From the calculated overlaps between the ZLL wavefunctions before and after the gap closure (not shown), we find that the higher- and lower-energy ZLLs are inverted at the gap closure. On the other hand, the size of the energy gap of the ZLLs near B increases monotonically with $B$ (Fig. $4 \mathrm{~b}$ ).

For comparison, we have calculated the Landau levels from a linear energy-vs-wavevector model devoid of coupling between Weyl points with opposite chiral charges (see Methods [26]). Regardless of the strength of the magnetic field, the ZLLs obtained from the low-energy theory exhibit gapless, linear energy-vs-wavevector dispersions and well-defined chiralities (Fig. 3). Also, the low-energy theory cannot properly describe the wavefunctions of the gapped ZLLs at high magnetic fields 
(Supplementary Fig. 3 [26]).

We find that the size of the energy gap is closely related to the distance between the two associated Weyl points along the direction perpendicular to the the applied magnetic field. (Note that the semiclassical electron orbit in momentum space is confined in the plane perpendicular to the magnetic field.) The two Weyl points at A are very close to each other (Fig. 2a) and the associated ZLLs can be easily mixed and gapped by a magnetic field along $x$ (Fig. 3d). Although there are four Weyl points at B (Fig. 2a), the four points are paired into two groups that are far away from each other in $k_{z}$ [2, 3], each consisting of two Weyl points with opposite chiralities that are very close together in the Brillouin zone (Fig. 2a). Thus the gap opening at B (Fig. 3e) can be understood similarly to the case of $\mathrm{A}$. The Weyl points at $\mathrm{C}$ are far away from each other in the Brillouin zone (Fig. 2a); hence, the associated ZLLs are not mixed, and the spectrum remains gapless (Fig. 3f).

It is tempting to conclude that a sizable energy gap opens up if the inverse magnetic length $l_{B}^{-1}=\sqrt{|e| B / \hbar}$, where $e$ is the charge of an electron and $\hbar$ is the reduced Planck constant, is comparable to the the distance in momentum space between the two Weyl points with opposite chiralities. To the contrary, the energy gap at a given magnetic field is much larger at $\mathrm{B}$ than at $\mathrm{A}$ (Fig. 4) although the distance between the Weyl points at B is longer than twice that at $\mathrm{A}$. We find that the electronic states with wavevectors whose distance from one Weyl point is within the distance between the corresponding opposite-chirality Weyl points (especially if their energies are close to the Weyl point energy) play an important role in the gap opening; hence, first-principles methods taking the full electronic structure into account without assuming any specific form of the low-energy Hamiltonian are useful (see Supplementary Fig. 3 and Supplementary Discussion 1 [26]).

For a comprehensive understanding of the subject matter, we have probed the dependence of the Landau levels on the direction of the magnetic field. If the magnetic field is applied along $x$ the Hamiltonian is invariant under $x \rightarrow-x$. Because of this mirror symmetry, the Landaulevel spectra at $k_{B}$ and $-k_{B}$ are the same (Figs. 5a and $5 \mathrm{~d})$. If the magnetic field is not along $x$, those spectra are not the same and can be significantly different (Figs. 5b, $5 \mathrm{c}, 5 \mathrm{e}$, and $5 \mathrm{f}$ ).

We have further studied other materials in TaAs family. Our calculations on NbAs, TaP, and NbP (Supplementary Figs. 4-6 [26]) show that the magnetic-fieldinduced chiral-symmetry breaking and gap opening generally occurs in Weyl semimetals.

The concept of chiral anomaly has been useful in understanding a variety of phenomena in Weyl semimetals. However, the gap opening in the zeroth Landau levels found in our study indicates the failure of the traditional picture of the chiral anomaly in Weyl semimetals in a strong magnetic field. Also, this change in the electronic structure of a Weyl semimetal induced by a magnetic field may lead to even more intriguing and richer physics. Among possible mechanisms such as strain that can break the chirality of Weyl fermions in condensed matter systems, magnetic field is unique in that it is an inherent element of the chiral magnetic effect initially conceived as the signature of chiral anomaly by Nielsen and Ninomiya 9 .

To the best of our knowledge, this work is the first $a b$ initio study on the Landau levels of real Weyl semimetals that takes account of the full electronic structure. The key findings of our study are beyond the description of the commonly adopted low-energy theories, in which the coupling between Weyl points by a magnetic field is assumed to be negligibly weak without justification. The powerful method used in our study can be applied in investigating the electromagnetic responses in other classes of novel semimetallic phases.

Note added: After we finished this work, we became aware of two independent experimental studies reporting that the longitudinal magnetoresistance of TaAs at a high-field regime increases with the strength of the magnetic field and becomes positive [30, 31. In Ref. 30, although the authors did not discuss the mechanism in detail in their preprint which does not have the supplementary information, they ascribed the experimental results to the gapping of the Weyl points at strong magnetic fields. After submission of our manuscript, a paper reporting an experimental discovery of a sharp sign reversal in the Hall resistivity of TaP at $B=35 \mathrm{~T}$ was published [32. The authors attributed the results to an energy gap opening at the Weyl points due to the magnetic tunneling at strong magnetic fields. They have introduced a $\mathbf{k} \cdot \mathbf{p}$ Hamiltonian which can describe the region in $\mathrm{k}$ space close to the Weyl points to explain their experimental data. Lastly, after this work was made public through posting as an e-print 33, two studies relevant to the breakdown of the chiral anomaly also became public as e-prints 34, 35. In Ref. 34, Chan and Lee reported that the anisotropy in group velocity near the Weyl point plays an important role in the hybridization and gapping between the zeroth Landau levels by using an effective Hamiltonian. Our results that the energy gap in Landau level spectrum is much larger at B than at A (Fig. 4) are consistent with their theory when applied to TaAs.

This work was supported by the Creative-Pioneering Research Program through Seoul National University.

\footnotetext{
* cheolhwan@snu.ac.kr

[1] X. Wan, A. M. Turner, A. Vishwanath, and S. Y. Savrasov, "Topological semimetal and Fermi-arc surface states in the electronic structure of pyrochlore iridates," Phys. Rev. B 83, 205101 (2011).
} 
[2] S.-M. Huang, S.-Y. Xu, I. Belopolski, C.-C. Lee, G. Chang, B. Wang, N. Alidoust, G. Bian, M. Neupane, C. Zhang, S. Jia, A. Bansil, H. Lin, and M. Z. Hasan, "A Weyl fermion semimetal with surface Fermi arcs in the transition metal monopnictide TaAs class," Nature Commun. 6, 7373 (2015).

[3] H. Weng, C. Fang, Z. Fang, B. A. Bernevig, and X. Dai, "Weyl semimetal phase in noncentrosymmetric transition-metal monophosphides," Phys. Rev. X 5, 011029 (2015)

[4] S.-Y. Xu, I. Belopolski, N. Alidoust, M. Neupane, G. Bian, C. Zhang, R. Sankar, G. Chang, Z. Yuan, C.C. Lee, S.-M. Huang, H. Zheng, J. Ma, D. S. Sanchez, B. Wang, A. Bansil, F. Chou, P. P. Shibayev, H. Lin, S. Jia, and M. Z. Hasan, "Discovery of a Weyl fermion semimetal and topological Fermi arcs," Science 349, 613617 (2015)

[5] B. Q. Lv, H. M. Weng, B. B. Fu, X. P. Wang, H. Miao, J. Ma, P. Richard, X. C. Huang, L. X. Zhao, G. F. Chen, Z. Fang, X. Dai, T. Qian, and H. Ding, "Experimental discovery of Weyl semimetal TaAs," Phys. Rev. X 5, 031013 (2015)

[6] S.-Y. Xu, N. Alidoust, I. Belopolski, Z. Yuan, G. Bian, T.-R. Chang, H. Zheng, V. N. Strocov, D. S. Sanchez, G. Chang, C. Zhang, D. Mou, Y. Wu, L. Huang, C.-C. Lee, S.-M. Huang, B. Wang, A. Bansil, H.-T. Jeng, T. Neupert, A. Kaminski, H. Lin, S. Jia, and M. Z. Hasan, "Discovery of a Weyl fermion state with Fermi arcs in niobium arsenide," Nature Phys. 11, 748-754 (2015).

[7] S.-Y. Xu, I. Belopolski, D. S. Sanchez, C. Zhang, G. Chang, C. Guo, G. Bian, Z. Yuan, H. Lu, T.-R. Chang, P. P. Shibayev, M. L. Prokopovych, N. Alidoust, H. Zheng, C.-C. Lee, S.-M. Huang, R. Sankar, F. Chou, C.-H. Hsu, H.-T. Jeng, A. Bansil, T. Neupert, V. N. Strocov, H. Lin, S. Jia, and M. Z. Hasan, "Experimental discovery of a topological Weyl semimetal state in TaP," Science Adv. 1, e1501092 (2015)

[8] Xu D.-F., Du Y.-P., Wang Z., Li Y.-P., Niu X.-H., Yao Q., Dudin P., Xu Z.-A., Wan X.-G., and Feng D.L., "Observation of Fermi arcs in non-centrosymmetric Weyl semi-metal candidate NbP," Chin. Phys. Lett. 32, 107101 (2015).

[9] H.B. Nielsen and M. Ninomiya, "The Adler-Bell-Jackiw anomaly and Weyl fermions in a crystal," Phys. Lett. B 130, $389-396$ (1983)

[10] S. L. Adler, "Axial-vector vertex in spinor electrodynamics," Phys. Rev. 177, 2426-2438 (1969).

[11] J. S. Bell and R. Jackiw, "A PCAC puzzle: $\pi^{0} \rightarrow \gamma \gamma$ in the $\sigma$-model," Il Nuovo Cimento A 60, 47-61 (1969).

[12] V. Aji, "Adler-Bell-Jackiw anomaly in Weyl semimetals: Application to pyrochlore iridates," Phys. Rev. B 85, 241101 (2012).

[13] A. A. Zyuzin and A. A. Burkov, "Topological response in Weyl semimetals and the chiral anomaly," Phys. Rev. B 86, 115133 (2012)

[14] D. T. Son and B. Z. Spivak, "Chiral anomaly and classical negative magnetoresistance of Weyl metals," Phys. Rev. B 88, 104412 (2013)

[15] S. A. Parameswaran, T. Grover, D. A. Abanin, D. A. Pesin, and A. Vishwanath, "Probing the chiral anomaly with nonlocal transport in three-dimensional topological semimetals," Phys. Rev. X 4, 031035 (2014).

[16] A. A. Burkov, "Chiral anomaly and diffusive magnetotransport in Weyl metals," Phys. Rev. Lett. 113, 247203
$(2014)$

[17] A. A. Burkov, "Negative longitudinal magnetoresistance in Dirac and Weyl metals," Phys. Rev. B 91, 245157 (2015)

[18] X. Huang, L. Zhao, Y. Long, P. Wang, D. Chen, Z. Yang, H. Liang, M. Xue, H. Weng, Z. Fang, $\mathrm{X}$. Dai, and G. Chen, "Observation of the chiralanomaly-induced negative magnetoresistance in 3D Weyl semimetal TaAs," Phys. Rev. X 5, 031023 (2015)

[19] C. Shekhar, A. K. Nayak, Y. Sun, M. Schmidt, M. Nicklas, I. Leermakers, U. Zeitler, Y. Skourski, J. Wosnitza, Z. Liu, Y. Chen, W. Schnelle, H. Borrmann, Y. Grin, C. Felser, and B. Yan, "Extremely large magnetoresistance and ultrahigh mobility in the topological Weyl semimetal candidate NbP," Nature Phys. 11, 645-649 (2015).

[20] C.-L. Zhang, S.-Y. Xu, I. Belopolski, Z. Yuan, Z. Lin, B. Tong, G. Bian, N. Alidoust, C.-C. Lee, S.-M. Huang, T.-R. Chang, G. Chang, C.-G. Hsu, G.-T. Jeng, M. Neupane, D. S. Sanchez, H. Zheng, J. Wang, H. Lin, C. Zhang, H.-Z. Lu, S.-Q. Shen, T. Neupert, M. Z. Hasan, and S. Jia, "Signatures of the Adler-Bell-Jackiw chiral anomaly in a Weyl fermion semimetal," Nature Commun. 7, 10735 (2016).

[21] F. Arnold, C. Shekhar, S.-C. Wu, Y. Sun, R. D. dos Reis, N. Kumar, M. Naumann, M. O. Ajeesh, M. Schmidt, A. G. Grushin, J. H. Bardarson, M. Baenitz, D. Sokolov, H. Borrmann, M. Nicklas, C. Felser, E. Hassinger, and B. Yan, "Negative magnetoresistance without welldefined chirality in the Weyl semimetal TaP," Nature Commun. 7, 11615 (2016).

[22] R. Lundgren, P. Laurell, and G. A. Fiete, "Thermoelectric properties of Weyl and Dirac semimetals," Phys. Rev. B 90, 165115 (2014).

[23] M. Hirschberger, S. Kushwaha, Z. Wang, Q. Gibson, S. Liang, C. A. Belvin, B. A. Bernevig, R. J. Cava, and N. P. Ong, "The chiral anomaly and thermopower of Weyl fermions in the half-heusler GdPtBi," Nature Mater. 15, 1161-1165 (2016).

[24] I. Panfilov, A. A. Burkov, and D. A. Pesin, "Density response in Weyl metals," Phys. Rev. B 89, 245103 (2014).

[25] J. Zhou, H.-R. Chang, and D. Xiao, "Plasmon mode as a detection of the chiral anomaly in Weyl semimetals," Phys. Rev. B 91, 035114 (2015).

[26] See Supplemental Material http://link.aps.org/ for more details of our calculations and the supporting results which include the crystal structure, electronic band structures, Landau-level states of TaAs, and Landaulevel spectra of $\mathrm{NbAs}$, TaP, and $\mathrm{NbP}$, which includes Refs. [36 58.

[27] R. Y. Chen, Z. G. Chen, X.-Y. Song, J. A. Schneeloch, G. D. Gu, F. Wang, and N. L. Wang, "Magnetoinfrared spectroscopy of Landau levels and Zeeman splitting of three-dimensional massless Dirac fermions in $\mathrm{ZrTe}_{5}$, , Phys. Rev. Lett. 115, 176404 (2015).

[28] P. J. W. Moll, A. C. Potter, N. L. Nair, B. J. Ramshaw, K. A. Modic, S. Riggs, B. Zeng, N. J. Ghimire, E. D. Bauer, R. Kealhofer, F. Ronning, and J. G. Analytis, "Magnetic torque anomaly in the quantum limit of Weyl semimetals," Nature Commun. 7, 12492 (2016).

[29] Y. Liu, X. Yuan, C. Zhang, Z. Jin, A. Narayan, C. Luo, Z. Chen, L. Yang, J. Zou, X. Wu, S. Sanvito, Z. Xia, L. Li, Z. Wang, and F. Xiu, "Zeeman splitting and dynamical mass generation in Dirac semimetal $\mathrm{ZrTe}_{5}$," Na- 
ture Commun. 7, 12516 (2016).

[30] Q. R. Zhang, B. Zeng, D. Rhodes, S. Memaran, T. Besara, R. Sankar, F. Chou, N. Alidoust, S.-Y. Xu, I. Belopolski, M. Z. Hasan, and L. Balicas, "Magnetic fieldinduced electronic and topological phase transitions in Weyl type-I semi-metals," , arXiv:1705.00920.

[31] B. J. Ramshaw, K. A. Modic, A. Shekhter, P. J. W. Moll, M. K. Chan, J. B. Betts, F. Balakirev, A. Migliori, N. J. Ghimire, E. D. Bauer, F. Ronning, and R.D. McDonald, "Annihilation of Weyl nodes in the extreme quantum limit of TaAs," , arXiv:1704.06944.

[32] C.-L. Zhang, S.-Y. Xu, C. M. Wang, Z. Lin, Z. Z. Du, C. Guo, C.-C. Lee, H. Lu, Y. Feng, S.-M. Huang, G. Chang, C.-H. Hsu, H. Liu, H. Lin, L. Li, C. Zhang, J. Zhang, X.-C. Xie, T. Neupert, M. Z. Hasan, H.-Z. Lu, J. Wang, and S. Jia, "Magnetic-tunnelling-induced Weyl node annihilation in TaP," Nature Phys. 13, 979 (2017)

[33] P. Kim, J. H. Ryoo, and C.-H. Park, "The chiral anomaly in Weyl semimetals is not robust," , arXiv:1707.01103.

[34] C.-K. Chan and P. A. Lee, "Emergence of gapped bulk and metallic side walls in the zeroth Landau level in Dirac and Weyl semimetals," Phys. Rev. B 96, 195143 (2017).

[35] D. R. Saykin, K. S. Tikhonov, and Ya. I. Rodionov, "Landau levels with magnetic tunnelling in Weyl semimetal and magnetoconductance of ballistic pn junction," , arXiv:1711.00684.

[36] P. Giannozzi, S. Baroni, N. Bonini, M. Calandra, R. Car, C. Cavazzoni, D. Ceresoli, G. L. Chiarotti, M. Cococcioni, I. Dabo, A. Dal Corso, S. de Gironcoli, S. Fabris, G. Fratesi, R. Gebauer, U. Gerstmann, C. Gougoussis, A. Kokalj, M. Lazzeri, L. Martin-Samos, N. Marzari, F. Mauri, R. Mazzarello, S. Paolini, A. Pasquarello, L. Paulatto, C. Sbraccia, S. Scandolo, G. Sclauzero, A. P. Seitsonen, A. Smogunov, P. Umari, and R. M. Wentzcovitch, "QUANTUM ESPRESSO: A modular and opensource software project for quantum simulations of materials," J. Phys.: Condens. Matt. 21, 395502 (2009).

[37] D. R. Hamann, "Optimized norm-conserving Vanderbilt pseudopotentials," Phys. Rev. B 88, 085117 (2013).

[38] M. Schlipf and F. Gygi, "Optimization algorithm for the generation of ONCV pseudopotentials," Comput. Phys. Commun. 196, $36-44$ (2015)

[39] J. P. Perdew, K. Burke, and M. Ernzerhof, "Generalized gradient approximation made simple," Phys. Rev. Lett. 77, 3865-3868 (1996).

[40] H. J. Monkhorst and J. D. Pack, "Special points for Brillouin-zone integrations," Phys. Rev. B 13, 5188-5192 (1976)

[41] H. Boller and E. Parthé, "The transposition structure of NbAs and of similar monophosphides and arsenides of niobium and tantalum," Acta Crystallogr. 16, 1095-1101 (1963)

[42] J.-O. Willerstrm, "Stacking disorder in NbP, TaP, NbAs and TaAs," J. Less Comm. Met. 99, 273 - 283 (1984).

[43] N. Marzari and D. Vanderbilt, "Maximally localized generalized Wannier functions for composite energy bands," Phys. Rev. B 56, 12847-12865 (1997)

[44] I. Souza, N. Marzari, and D. Vanderbilt, "Maximally localized Wannier functions for entangled energy bands," Phys. Rev. B 65, 035109 (2001)

[45] A. A. Mostofi, J. R. Yates, Y.-S. Lee, I. Souza, D. Vanderbilt, and N. Marzari, "Wannier90: A tool for obtaining maximally-localised Wannier functions," Comput. Phys. Commun. 178, $685-699$ (2008)
[46] N. Marzari, A. A. Mostofi, J. R. Yates, I. Souza, and D. Vanderbilt, "Maximally localized Wannier functions: Theory and applications," Rev. Mod. Phys. 84, 14191475 (2012).

[47] R. Peierls, "Zur Theorie des Diamagnetismus von Leitungselektronen," Zeitschrift für Physik 80, 763-791 (1933)

[48] S. Ismail-Beigi, E. K. Chang, and S. G. Louie, "Coupling of nonlocal potentials to electromagnetic fields," Phys. Rev. Lett. 87, 087402 (2001).

[49] Z.-M. Yu, Y. Yao, and S. A. Yang, "Predicted unusual magnetoresponse in type-II Weyl semimetals," Phys. Rev. Lett. 117, 077202 (2016)

[50] M. Udagawa and E. J. Bergholtz, "Field-selective anomaly and chiral mode reversal in type-II Weyl materials," Phys. Rev. Lett. 117, 086401 (2016)

[51] S. Tchoumakov, M. Civelli, and M. O. Goerbig, "Magnetic-field-induced relativistic properties in typeI and type-II Weyl semimetals," Phys. Rev. Lett. 117, 086402 (2016).

[52] A. A. Soluyanov, D. Gresch, Z. Wang, Q. Wu, M. Troyer, X. Dai, and B. A. Bernevig, "Type-II Weyl semimetals," Nature 527, 495-498 (2015).

[53] G. Autès, D. Gresch, M. Troyer, A. A. Soluyanov, and O. V. Yazyev, "Robust type-II Weyl semimetal phase in transition metal diphosphides $X \mathrm{P}_{2}(X=\mathrm{Mo}, \mathrm{W})$," Phys. Rev. Lett. 117, 066402 (2016)

[54] R. Yu, H. Weng, Z. Fang, H. Ding, and X. Dai, "Determining the chirality of Weyl fermions from circular dichroism spectra in time-dependent angle-resolved photoemission," Phys. Rev. B 93, 205133 (2016).

[55] H. Huang, S. Zhou, and W. Duan, "Type-II Dirac fermions in the $\mathrm{PtSe}_{2}$ class of transition metal dichalcogenides," Phys. Rev. B 94, 121117 (2016).

[56] D. Gresch, Q. Wu, G. W. Winkler, and A. A. Soluyanov, "Hidden Weyl points in centrosymmetric paramagnetic metals," New J. Phys. 19, 035001 (2017).

[57] X. Wang, J. R. Yates, I. Souza, and D. Vanderbilt, " $A b$ initio calculation of the anomalous Hall conductivity by Wannier interpolation," Phys. Rev. B 74, 195118 (2006).

[58] J. R. Yates, X. Wang, D. Vanderbilt, and I. Souza, "Spectral and Fermi surface properties from Wannier interpolation," Phys. Rev. B 75, 195121 (2007) 


\section{Supplemental Material: Breakdown of the Chiral Anomaly in Weyl Semimetals in a Strong Magnetic Field}

\section{Methods}

Electronic-structure calculations. Electronic structures were calculated within the framework of density functional theory as implemented in the Quantum-ESPRESSO package [36]. Spin-orbit coupling effects were treated self-consistently using fullyrelativistic, norm-conserving pseudopotentials [37, 38. The exchange-correlation energy was approximated by the scheme of Perdew, Burke, and Ernzerhof [39]. The kinetic energy cutoff was set to $100 \mathrm{Ry}$, and the Brillouin zone was sampled with a $12 \times 12 \times 8$ MonkhostPack 40] k-point mesh. The experimental lattice parameters [2, 7, 41, 42] were used.

$A b$ initio tight-binding model. We constructed maximally-localized Wannier functions using the $d$ orbitals of $\mathrm{Ta}$ or $\mathrm{Nb}$ atoms and $p$ orbitals of $\mathrm{As}$ or $\mathrm{P}$ atoms as an initial guess 43 46. We then obtained the Hamiltonian matrix elements between the Wannier orbitals. We incorporated the effect of a magnetic field into the tight-binding Hamiltonian via the Peierls substitution [7],

$$
t_{i j} \rightarrow t_{i j} \exp \left[i \frac{e}{\hbar} \int_{\mathbf{r}_{i}}^{\mathbf{r}_{j}} \mathbf{A} \cdot d \mathbf{l}\right]
$$

where $e$ is the charge of an electron, $\hbar$ is the reduced Planck constant, $t_{i j}$ is the Hamiltonian matrix element between the Wannier orbitals $i$ and $j$ in the absence of an external magnetic field, and $\mathbf{A}=\left(B_{y} z,-B_{x} z, 0\right)$ is the vector potential that corresponds to the magnetic field $\mathbf{B}=\left(B_{x}, B_{y}, 0\right)$. The integration was performed along a straight line between the Wannier centers $\mathbf{r}_{i}$ and $\mathbf{r}_{j}$ following the theory of Ref. [48. We have checked that our computational results do not depend on the gauge of the vector potential. As the additional phase in Eq. (1) breaks the translational symmetry, we employed the supercell method. Nielsen and Ninomiya 9 and the recent studies on the Landau levels of Weyl semimetals 4951 have neglected the effects of Zeeman splitting because (i) the orbital effects are more important 49 51] and, (ii) much more importantly, the Zeeman splitting merely induces a shift in the positions of the Weyl points in the Brillouin zone and hence results only in quantitative changes and all the qualitative findings in those studies [9, 49,51] and in ours remain valid.

Ab initio $2 \times 2$ model. Near a Weyl point, the effective, low-energy Hamiltonian at wavevector $\mathbf{k}$ can be written as $H(\mathbf{k})=\sum_{i=1}^{3} \hbar k_{i} V_{i} I+\sum_{i, j=1}^{3} \hbar k_{i} A_{i j} \sigma_{j}$, where $I$ is the two-dimensional identity matrix and $\sigma$ 's are the Pauli matrices. Previous studies have determined the parameters in the effective Hamiltonian to best reproduce the $a b$ initio electronic band structure near the Weyl point 52 56. In our study, we took advantage of the matrix elements of the velocity operator right at the Weyl point between the doubly-degenerate eigenstates 57, 58. The velocity matrix elements are written as $v_{n m, i}=\left\langle\psi_{n}\left|\hat{v}_{i}\right| \psi_{m}\right\rangle$, where $\hat{v}_{i}$ is the $i$-th component of the velocity operator, $m$ and $n$ are the band indices, and $\left|\psi_{1}\right\rangle$ and $\left|\psi_{2}\right\rangle$ are the degenerate Bloch states at the Weyl point. In terms of the velocity matrix elements, $V_{i}$ and $A_{i j}$ are given by

$$
\begin{aligned}
V_{i} & =\left(v_{11, i}+v_{22, i}\right) / 2 \\
A_{i 1} & =\operatorname{Re}\left[v_{12, i}\right] \\
A_{i 2} & =-\operatorname{Im}\left[v_{12, i}\right] \\
A_{i 3} & =\left(v_{11, i}-v_{22, i}\right) / 2 .
\end{aligned}
$$

Unlike the previous methods based on ab initio electronic band structures [52 56], our method naturally determines the sign of the chiral charge of a Weyl point, which is the sign of the determinant of matrix $A$. The effect of the magnetic field was incorporated into the Hamiltonian $H(\mathbf{k})$ by the minimal coupling, $H(\mathbf{k}) \rightarrow H\left(\mathbf{k}-\frac{e}{\hbar} \mathbf{A}\right)$. For numerical calculations, we employed the supercell method as in the $a b$ initio tight-binding model. 


\section{Supplementary Discussion 1: Discussion on the results shown in Supplementary Fig. 3}

There are two pronounced peaks in the density of states near the Weyl point B only in the full ab-initio tight-binding calculations but not in ab initio $2 \times 2$ model calculations (Supplementary Fig. 3d). From perturbation theory, nearby Bloch states with similar energies as $E^{\mathrm{W}}$ strongly mix by the magnetic field and form Landaulevel states. We emphasize that in order to describe this mixing correctly, $a b$ initio calculations taking into account the full electronic states should be used because the states whose Bloch wavevectors are within the range of the separation between the two opposite-chirality Weyl points are all mixed together by an external magnetic field and are of crucial importance in forming the Landau levels and in opening up the band gap at the zeroth Landau levels. In our case, the distance of the van Hove singularities from the Weyl point at $\mathrm{B}\left(\sim 0.065 \AA^{-1}\right)$ (Supplementary Fig. 3b) is less than the separation of the two opposite-chirality Weyl points $\left(=0.072 \AA^{-1}\right)$. Therefore the van Hove singularity at energy $E^{\mathrm{W}}$ (Supplementary Figs. 3b and 3d) plays an important role in the gap opening.

The projection $P_{-}\left(k_{z}\right)$ from the ab initio tight-binding model at $\mathrm{B}$ is sizable near the van Hove singularities (Supplementary Fig. 3g). As we discussed above, the van-Hove-singularity states indeed participate in forming the zeroth-Landau-level states. Note that the $a b$ initio $2 \times 2$ model cannot properly describe the wavefunctions of the zeroth Landau levels if gapped (Supplementary Figs. 3f-3h; also see Fig. 3 of the main manuscript).

We conclude by emphasizing the importance of handling the full electronic states whose wavevector is within the range of the separation between the two oppositechirality Weyl points in describing this gap-opening physics. Our proposed $a b$ initio tight-binding method is well suited for this purpose. 
a

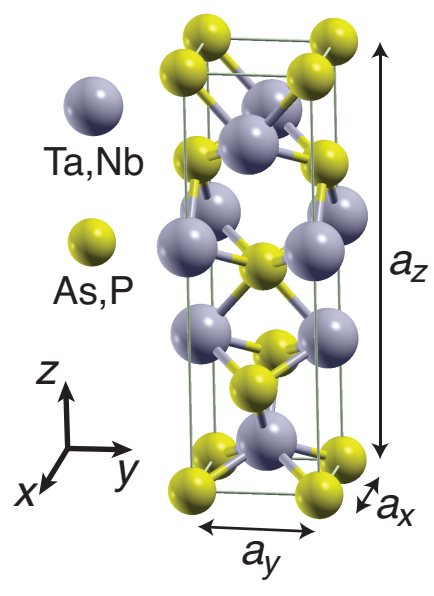

C

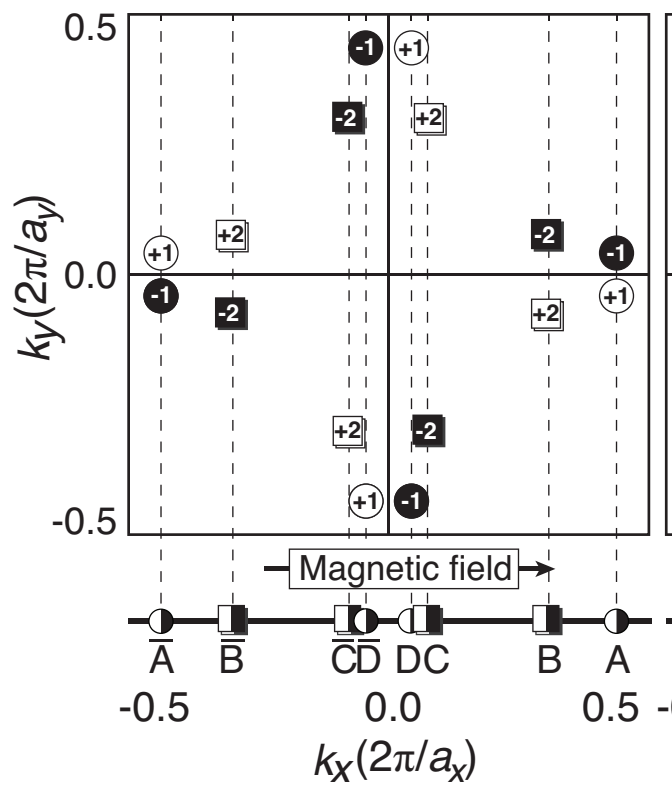

b

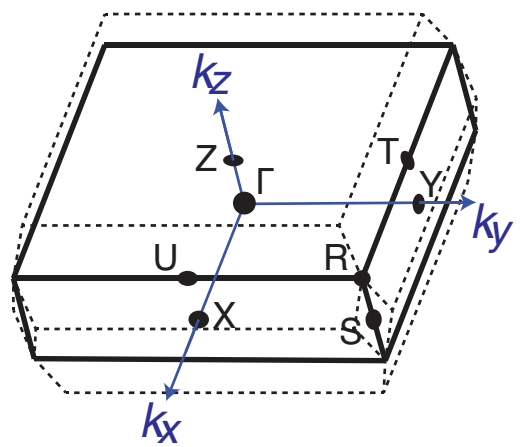

d $\quad \mathrm{NbAs}, \mathrm{NbP}$

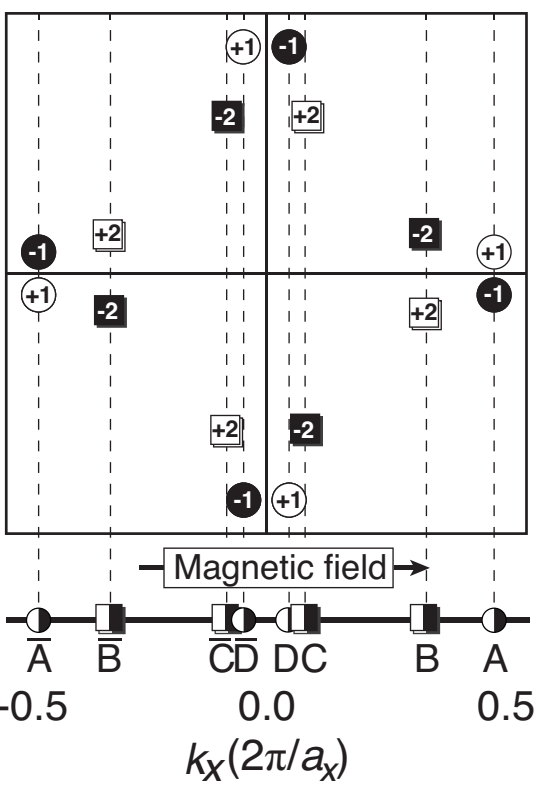

FIG. S1. Crystal structure and Brillouin zone of TaAs, NbAs, TaP, and NbP. a, Crystal structure of TaAs, NbAs, $\mathrm{TaP}$, and NbP. $a_{x}, a_{y}$, and $a_{z}$ are the lattice constants of the tetragonal (conventional) unit cell along the $x, y$, and $z$ directions $\left(a_{x}=a_{y}\right)$, respectively. $\mathbf{b}$, Brillouin zone of TaAs, NbAs, TaP, and NbP. The Brillouin zone of the tetragonal (conventional) unit cell, which is used in the computations, is drawn in thick solid lines. The Brillouin zone of the body-centered tetragonal (primitive) unit cell is drawn in thin dashed lines. c and $\mathbf{d}$, A schematic (not to scale) of the projected Weyl points and their chiral charges (enclosed numbers) of TaAs or TaP (c) and NbAs or NbP (d). 
a TaAs electronic band structure (tetragonal unit cell)

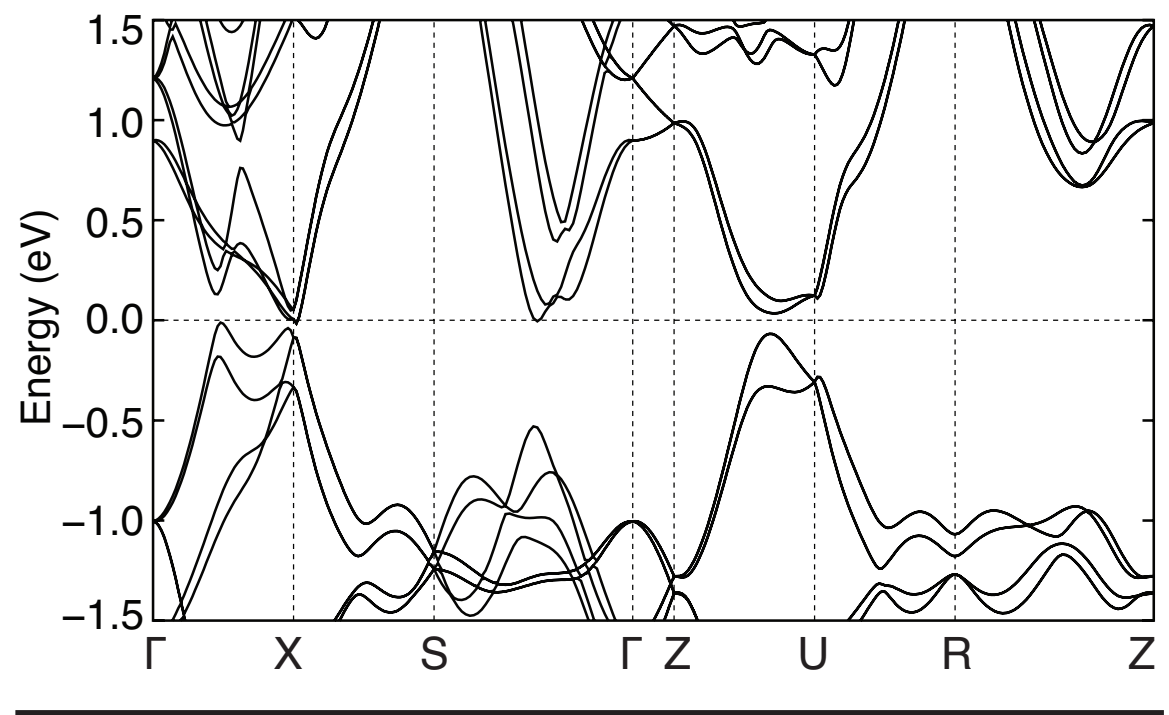

Electronic band structures around the Weyl points, W1 and W2

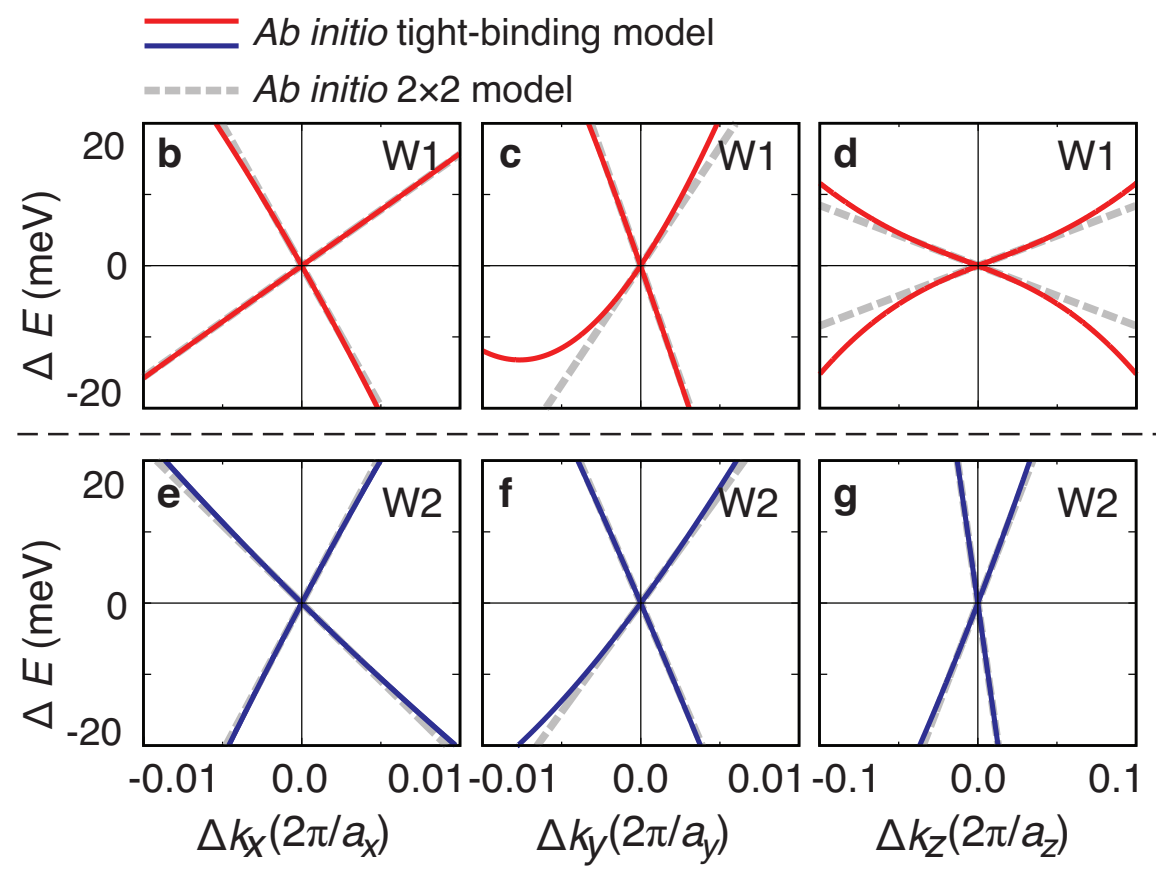

FIG. S2. Electronic band structures of TaAs. a, Electronic band structure along the symmetry lines of TaAs shown in Supplementary Fig. 1. The zero of energy is at the Fermi level. b-g, Electronic band structures along $k_{x}(\mathbf{b}$ and $\mathbf{e}), k_{y}(\mathbf{c}$ and $\mathbf{f})$, and $k_{z}(\mathbf{d}$ and $\mathbf{g})$. The origins of wavevector are set at the Weyl points, $\mathrm{W}_{1}\left(0.4879 \cdot 2 \pi / a_{x}, 0.0077 \cdot 2 \pi / a_{y}, 0\right)(\mathbf{b}-\mathbf{d})$ and $\mathrm{W}_{2}\left(0.2812 \cdot 2 \pi / a_{x}, 0.0197 \cdot 2 \pi / a_{y}, 0.4067 \cdot 2 \pi / a_{z}\right)(\mathbf{e}-\mathbf{g})$. The zeros of energy are set at the energies of the corresponding Weyl points. Solid red or solid blue curves represent the computational results obtained from the $a b$ initio tight-binding model. Dashed grey curves show the results from the $a b$ initio $2 \times 2$ model. 


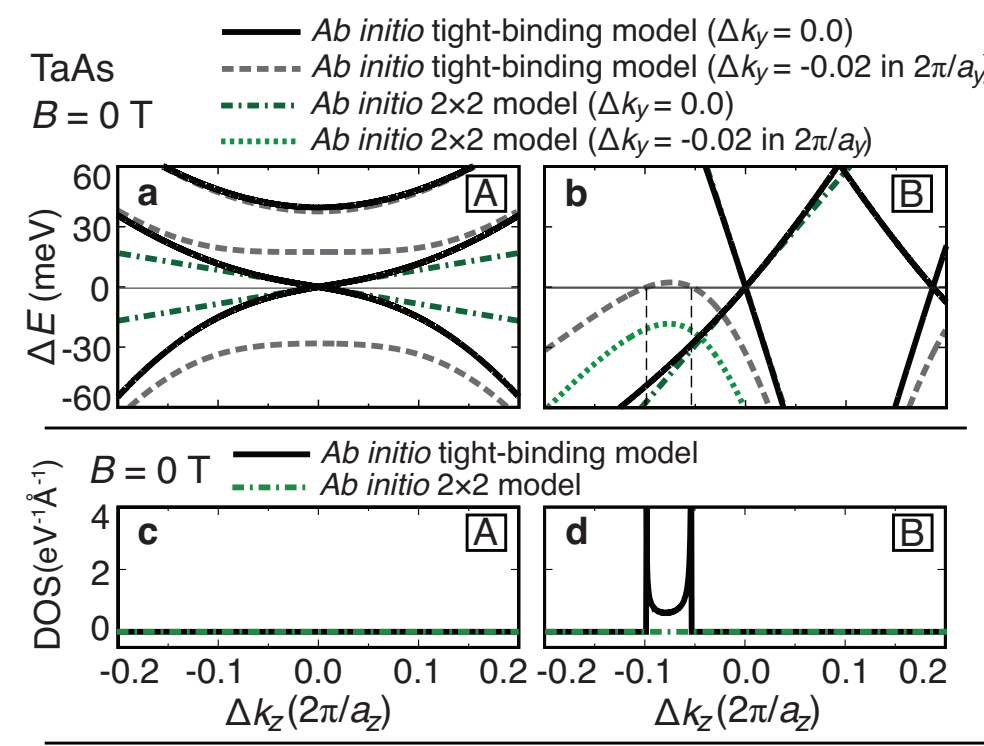

\begin{tabular}{ll}
\hline TaAs & Ab initio tight-binding model (Higher-energy 0th Landau level) \\
Zeroth Landau levels & $=---A b$ initio tight-binding model (Lower-energy $0^{\text {th }}$ Landau level) \\
& $----A b$ initio $2 \times 2$ model
\end{tabular}

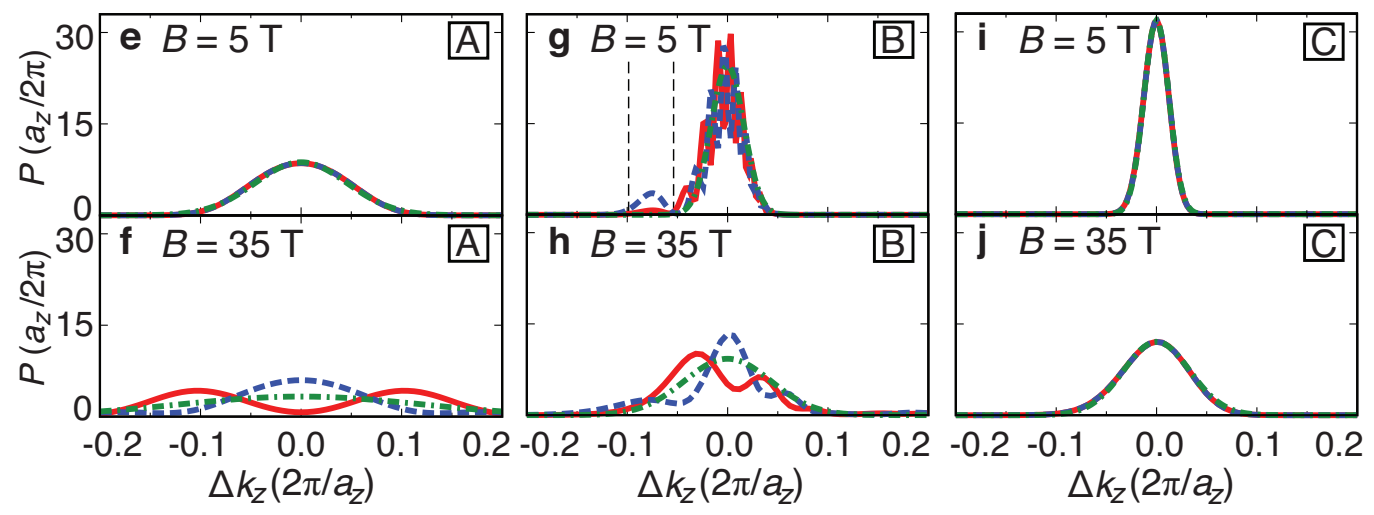

FIG. S3. Zeroth-Landau-level states of TaAs. a and b, Electronic band structures along $k_{z}$ for two different $k_{y}$ values. The origins of wavevector are set at the Weyl points, $\mathrm{W}_{1}(\mathbf{a})$ and $\mathrm{W}_{2}$ (b) (see the caption of Supplementary Fig. 2). The zeros of energy are set at the energies of the corresponding Weyl points. Solid black or dashed grey curves show the results from the $a b$ initio tight-binding model. Dash-dotted dark green and dotted green curves show the results from the $a b$ initio $2 \times 2$ model. c and $\mathbf{d}$, The $k_{z}$-resolved density of states whose energy and $k_{x}$ are the same as those of the Weyl points at W $\mathrm{W}_{1}(\mathbf{c})$ and $\mathrm{W}_{2}(\mathbf{d}): \operatorname{DOS}\left(k_{z}\right)=\frac{1}{2 \pi} \sum_{n} \int_{\mathrm{BZ}} d \mathbf{k}^{\prime} \delta\left(E_{n, \mathbf{k}^{\prime}}-E^{\mathrm{W}}\right) \delta\left(k_{x}^{\prime}-k_{x}^{\mathrm{W}}\right) \delta\left(k_{z}^{\prime}-k_{z}\right)$ where the $E_{n, \mathbf{k}^{\prime}}$ is the energy of the Bloch states at wavevector $\mathbf{k}^{\prime}$ with band index $n, E^{\mathrm{W}}$ and $k_{x}^{\mathrm{W}}$ are the energy and $k_{x}$ of the Weyl point, respectively, the integration is carried out over the Brillouin zone, and the summation runs over all band indices $n$. The dashed black lines in $\mathbf{b}$ and $\mathbf{g}$ are guides to the eye indicating the van Hove singularities. e-j, The projection $P$ of the zeroth-Landau-level states at A (e and $\mathbf{f})$, B (g and $\mathbf{h})$, and $\mathrm{C}(\mathbf{i}$ and $\mathbf{j})$ at $B=5 \mathrm{~T}(\mathbf{e}, \mathbf{g}$, and $\mathbf{i})$ and $B=35 \mathrm{~T}(\mathbf{f}, \mathbf{h}$, and $\mathbf{j})$ onto the Bloch states in the absence of a magnetic field. The projection $P$ is given by $P_{ \pm}\left(k_{z}\right)=\frac{2 \pi}{a_{z}} \sum_{n}\left|\left\langle\psi_{n, k_{x}}^{\mathrm{W}}\left(k_{z}\right) \mid \psi_{ \pm, k_{x}^{\mathrm{W}}}^{\mathrm{L}}\right\rangle\right|^{2}$ where $\left|\psi_{+}^{\mathrm{L}}\right\rangle$ and $\left|\psi_{-}^{\mathrm{L}}\right\rangle$ are the higher- and lower-energy zeroth-Landau-level states, respectively, and $|\psi\rangle$ is the Bloch state in the absence of a magnetic field. The summation runs over all band indices of the Bloch states $n$, and $k_{x}^{\mathrm{W}}$ is the $k_{x}$ component of the corresponding Weyl point. Solid red and dashed blue curves show $P_{+}\left(k_{z}\right)$ and $P_{-}\left(k_{z}\right)$, respectively, obtained from the ab initio tight-binding model. Dash-dotted green curves show the average of $P_{+}\left(k_{z}\right)$ and $P_{-}\left(k_{z}\right)$ obtained from the ab initio $2 \times 2$ model; we took their average because the two zeroth-Landau-level states are degenerate. The origins of $k_{z}$ are the $k_{z}$ components of $\mathrm{W}_{1}(\mathbf{e}$ and $\mathbf{f})$ and $\mathrm{W}_{2}(\mathbf{g}-\mathbf{j})$. 

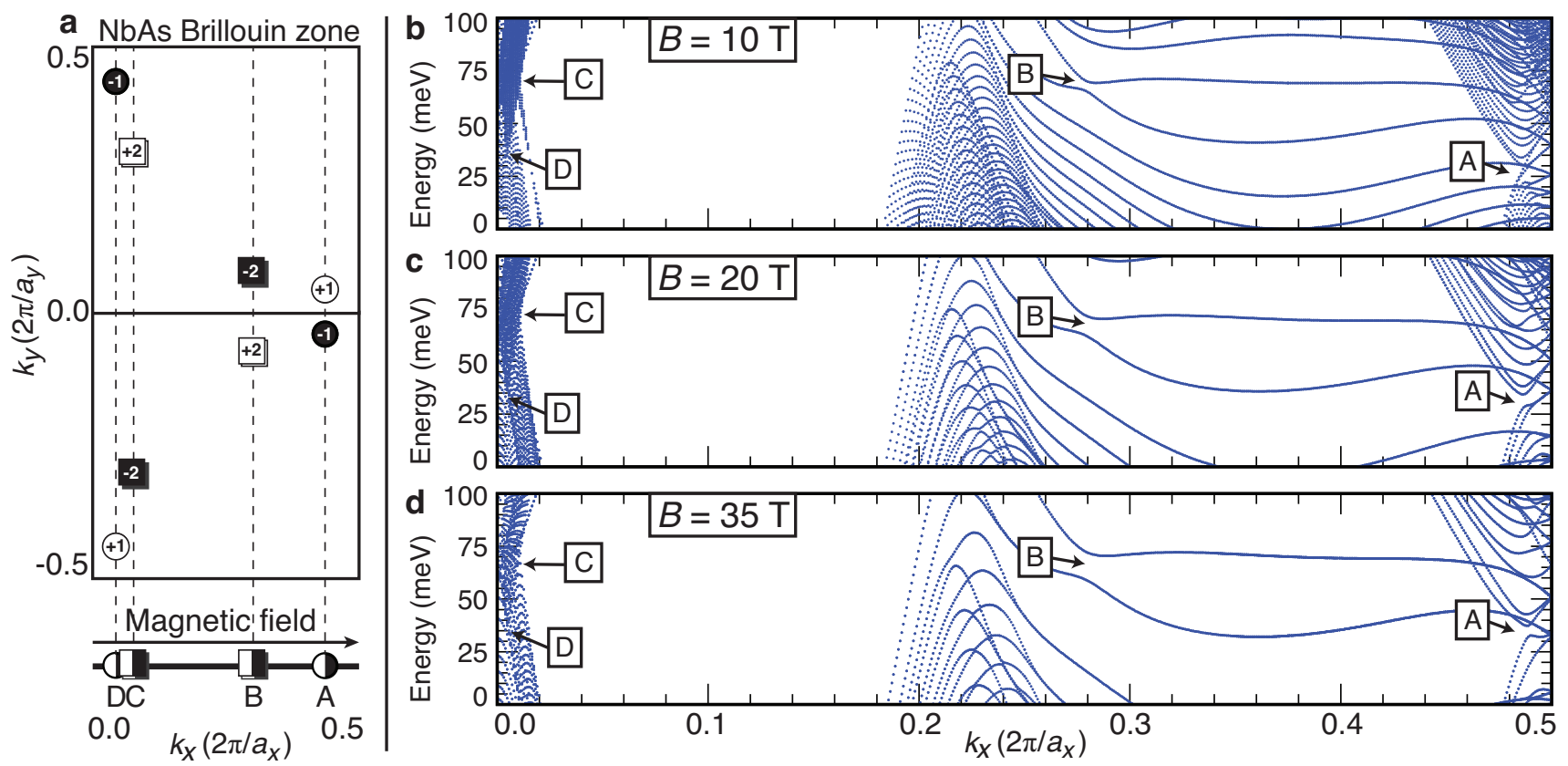

FIG. S4. Landau levels of NbAs. a, A schematic (not to scale) of the projected Weyl points and their chiral charges (the enclosed numbers). Half of the Brillouin zone $\left(k_{x}>0\right)$ is shown. Discs and squares represent two different classes of Weyl points. b-d, Landau-level spectra of NbAs in a magnetic field applied along $x$ obtained from $a b$ initio tight-binding calculations. The zero of energy is set at the Fermi level before the application of the magnetic field. A, B, C, and D denote the corresponding Weyl points shown in a.
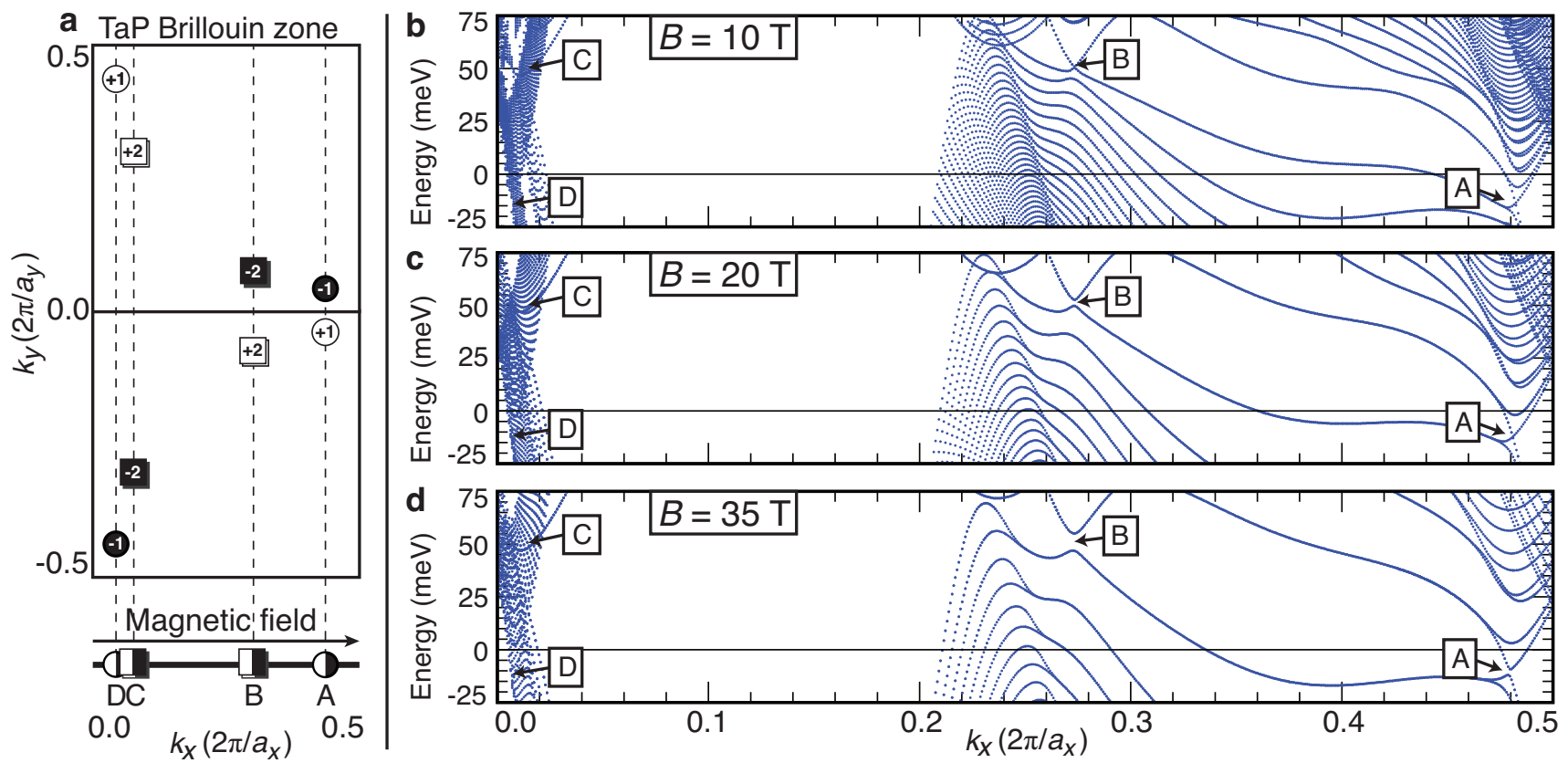

FIG. S5. Landau levels of TaP. a, A schematic (not to scale) of the projected Weyl points and their chiral charges (the enclosed numbers). Half of the Brillouin zone $\left(k_{x}>0\right)$ is shown. Discs and squares represent two different classes of Weyl points. b-d, Landau-level spectra of TaP in a magnetic field applied along $x$ obtained from ab initio tight-binding calculations. The zero of energy is set at the Fermi level before the application of the magnetic field. A, B, C, and D denote the corresponding Weyl points shown in a. 

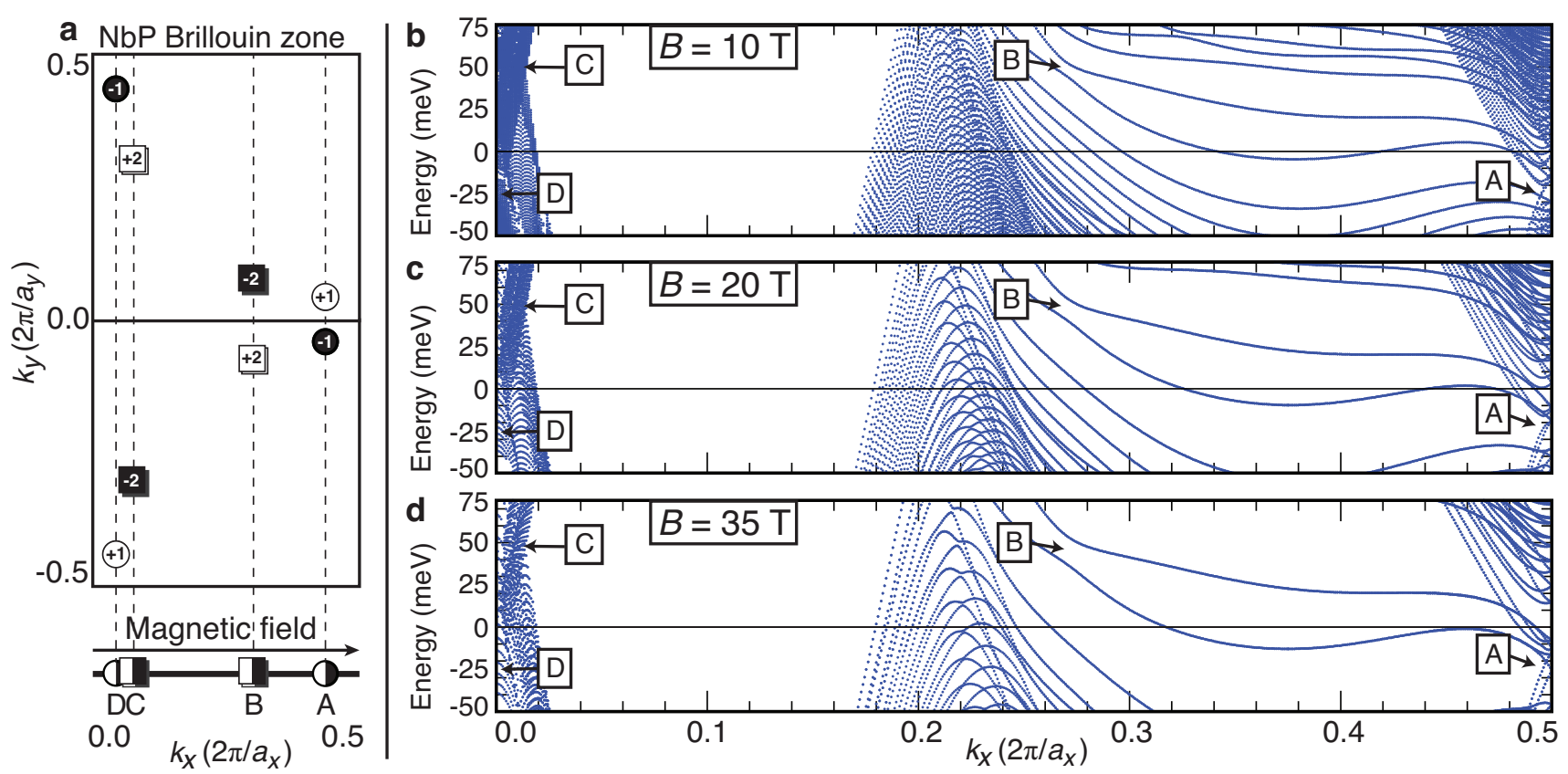

FIG. S6. Landau levels of NbP. a, A schematic (not to scale) of the projected Weyl points and their chiral charges (the enclosed numbers). Half of the Brillouin zone $\left(k_{x}>0\right)$ is shown. Discs and squares represent two different classes of Weyl points. b-d, Landau-level spectra of NbP in a magnetic field applied along $x$ obtained from $a b$ initio tight-binding calculations. The zero of energy is set at the Fermi level before the application of the magnetic field. A, B, C, and D denote the corresponding Weyl points shown in a. 